
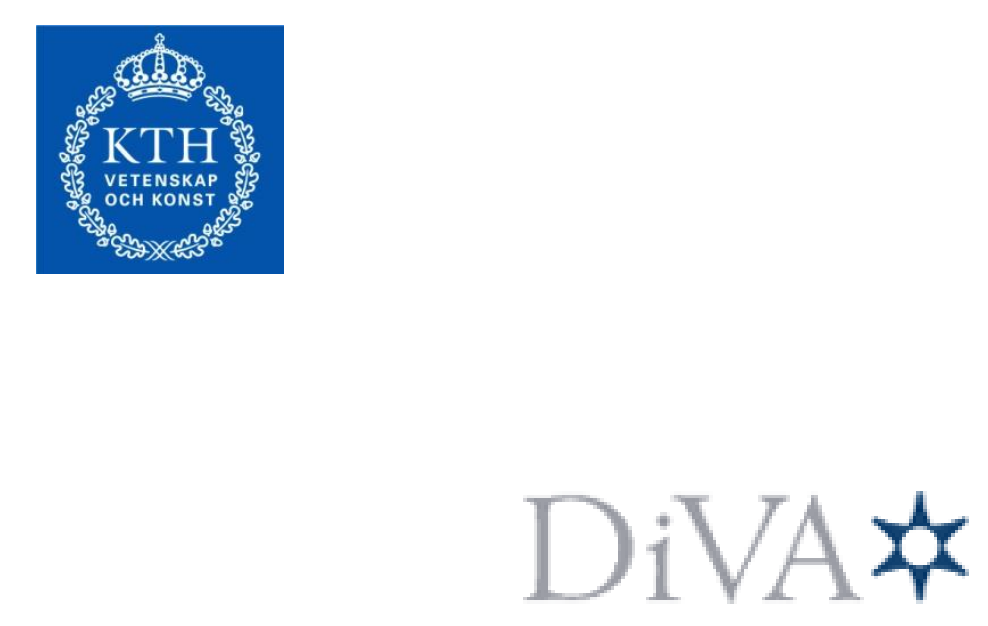

http://kth.diva-portal.org

This is an author produced version of a paper published in Proceedings of the 5th International Conference on Critical Infrastructure, CRIS 2010.

This paper has been peer-reviewed but does not include the final publisher proofcorrections or proceedings pagination.

(C) 2010 IEEE. Personal use of this material is permitted. Permission from IEEE must be obtained for all other uses, in any current or future media, including reprinting/republishing this material for advertising or promotional purposes, creating new collective works, for resale or redistribution to servers or lists, or reuse of any copyrighted component of this work in other works.

Citation for the published paper:

Moustafa Chenine, Iyad Al Khatib, Jordan Ivanovski, Volkan Maden, Lars Nordström.

PMU traffic shaping in IP-based wide area communication Proceedings of the 5th International Conference on Critical Infrastructure, CRIS 2010. Access to the published version may require subscription. Published with permission from: IEEE 


\title{
PMU Traffic Shaping in IP-Based Wide Area Communication
}

\author{
Moustafa Chenine, Student Member, IEEE, Iyad Al Khatib, Jordan Ivanovski, Volkan Maden, Lars \\ Nordström, Member, IEEE
}

\begin{abstract}
Phasor based Wide Area Monitoring and Control (WAMC) Systems are becoming a reality with increased research, development and deployments. Many potential control applications based on these systems are being proposed and researched. Such applications are either local applications using data from one or a few Phasor Measurement Units (PMUs) or centralized utilizing data from several PMUs. An aspect of these systems, which is less well researched, is the WAMC system dependence on high performance communication. This paper presents results of research on the use of Internet Protocol (IP) networks for both PMU and Remote Terminal Unit (RTU)/Intelligent Electronic Devices (IED) data for monitoring purposes. The paper examines performance aspects of the IP network Infrastructures when utilized by both: continuous PMU data streams and critical IED/RTU data. A set of simulation models characterizing a network of a Nordic Transmission System Operator were built to perform the analysis. The paper considers Quality of Service $(\mathrm{QoS})$ strategies and the resulting delays and data loss that would be experienced.
\end{abstract}

Index Terms-PMU Communication, Wide Area Monitoring and Control, communication simulation, PMU communication delays.

\section{INTRODUCTION}

$\mathrm{E}^{\mathrm{s}}$ LECTRICAL power networks are a part of the critical infrastructure in modern society. Our dependence and demand on electricity has risen sharply while recently this rising demand for electricity has been met with a serious strain in terms of production and expansion of transmission capacity. This is, among other factors, due to increasing environmental policies and costs. Furthermore, the re-regulation of the electrical market and the connection of national grids with neighbouring nations have resulted in a more complex and dynamic environment, in which multiple organizations coordinate and cooperate in the operation and control of the power system.

There is an international interest and implementation drive, in both academia and industry, on the prospects of PMU based monitoring and control technology [1], [2]. These systems promise to offer more accurate and timely data on the state of the power system increasing the possibilities to manage the

Moustafa Chenine and Lars Nordström are with the department of Industrial Information \& Control Systems, School of Electrical Engineering, The Royal Institute of Technology (KTH), Stockholm, SWEDEN. (phone: +46-8-790 6831; e-mail :\{moustafac | larsn\}@ics.kth.se). Iyad Al Khatib is at the Department of Signal Theory and Communications, Polytechnic University of Catalonia (UPC), Barcelona, Spain and with Solidux Telecom AB, Stockholm, Sweden (iyad@soliduxtelecom.com) system at a more efficient and responsive level and apply wide area control and protection schemes.

Generally, most of the international effort (e.g. the work published in [1], [2] ,[3], [4], and [5]) is on the development of monitoring and assessment application based on PMU measurements, in addition to platforms that would support these applications such as the Gridstat project [6]. Monitoring and assessment applications are known as Wide Area Monitoring Systems (WAMS) These new applications were not possible earlier with Supervisory Control And Data Acquisition (SCADA) measurements due to their generally low data sampling rate quality and lack of exact time synchronization. There has been less work on developing protection systems for PMU based monitoring and assessment application, and even less so for wide area control applications. The latter groups of systems do more than just monitoring the power system states, and they are referred to as Wide Area Monitoring and Control (WAMC) Systems.

\section{A. Purpose}

Quality of Service (QoS) in terms of guaranteeing delay limits and data completeness (realized by minimizing communication packet loss) is a crucial parameter for the success of communication networks for critical power infrastructure control systems. Hence, care must be taken in defining the performance level parameters required to ensure a satisfactory behavior of the network and protocols. The TCP/IP suite does not by itself guarantee the level of performance since it is a "best effort" protocol suite. On the other hand QoS mechanisms are available for IP-networks that allow the differentiation and prioritization of critical communication traffic, and thereby furthering performance requirements. This paper discusses these issues and presents results of simulations in which QoS mechanisms are applied to communication networks for Wide Area Monitoring using PMUs.

\section{B. Outline}

The rest of the paper is structured in five sections. Section II presents an overview of WAMC architectures outlining the main components of such systems; this section also discusses different communication paradigms and issues related to IP communication. Section III describes the simulation model scenarios that are built and the respective varied parameters. The analysis of the results of the simulation and a discussion are in section IV. Finally, section V concludes the paper. 


\section{GENERAL WAMC COMMUNICATION REQUIREMENTS}

A complete PMU based Monitoring and Control Systems is a system in which PMU measurements are collected from various locations in the electrical grid in a nation or region. The measurements are then communicated to a central location, where they are used by an assessment or monitoring application that would raise alarms or calculate results. The alarms raised and results calculated by these monitoring systems are- in turn- used to provide corrective actions or control on the power grid. Alternative architectures, such as using few remote PMU signals in a local system for a specific protection application is also possible, but are outside the scope of this study.

\section{A. WAMC Components}

A WAMC system includes four basic components: a PMU, a Phasor Data Concentrator, a PMU-based application system, and a communication network [7].

Logically, there are three layers in a WAMC, which in essence are very similar to the traditional SCADA systems. Layer 1 is the Data Acquisition layer, and it is where the WAMC system interfaces with the power system on substation bars and power lines. The PMUs are place in layer 1. Layer 2 is the Data Management layer, and it is where the PMU measurements are collected and sorted into a single time synchronized dataset. The last layer is Layer 3, which is the Application Layer. Layer 3 represents the real time PMU based application functions that process the time synchronized PMU measurements provided by Layer 2. Figure 1 illustrates the logical architecture of WAMC systems.

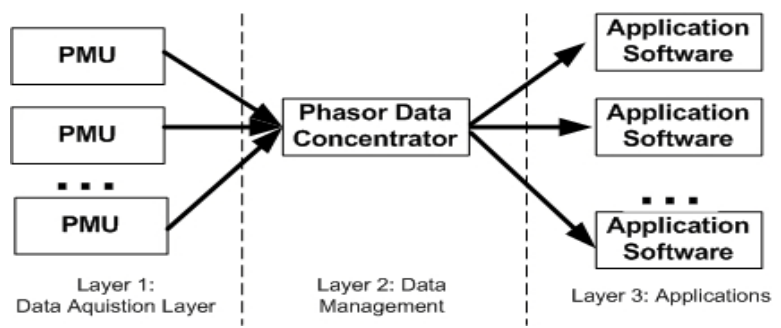

Figure 1: Layers and components of WAMC system.

\section{B. Communication Infrastructure}

The communication infrastructure is an important component in the architecture of a WAMC system. This is because PMU devices are distributed over a wide area, covering various locations within the boundary of a power system. The PMU devices are then connected to one or many control centers over the communication network. Therefore, the communication network constitutes a possible bottleneck in the architecture of these systems especially that delays and data quality of the remote signals from PMUs depend on the communication infrastructure.

\section{1) Dedicated network solutions}

Much of the previous work suggests the use of dedicated channels in optical fiber networks as the main communication media for PMU communication networks [8], [9]. The network architecture would then be composed of a main optical fiber backbone connected to the substation router. In turn, the PMU can be connected to the substation router. The PMU would be connected with the substation router through the standard substation Local Area Network (LAN). The measured phase angles from the PMUs are then transferred to a PDC, which sorts the phasors according to the GPS time stamps. The application buffer performs error and synchronization checking among other functionalities [7].

The main argument for using dedicated channels is for minimizing the effects of delay and congestion. Delay is an important measure for the success of PMU based application, since the main advantage of such application over SCADA based applications is the ability to observe transient phenomena in the power system. These phenomena could then be monitored directly in real time and used for control and protection purposes rather than merely for post disturbance analysis.

\section{2) Shared networks solutions}

As an alternative to the "dedicated network" solution, a wide area network based on TCP/IP protocol can be used. In such a network (see Figure 2), the traffic from PMUs is accompanied with traffic from substation RTUs. There are also other network uses for this scenario such as utility Voiceover-IP. The TCP/IP utilization in utility networking is fairly new, and it has been very well tested for wide area networks in this regard. Moreover TCP/IP offers an open standard and has wide adoption in industry. This means cheaper, more flexible, and interoperable software and hardware [10].

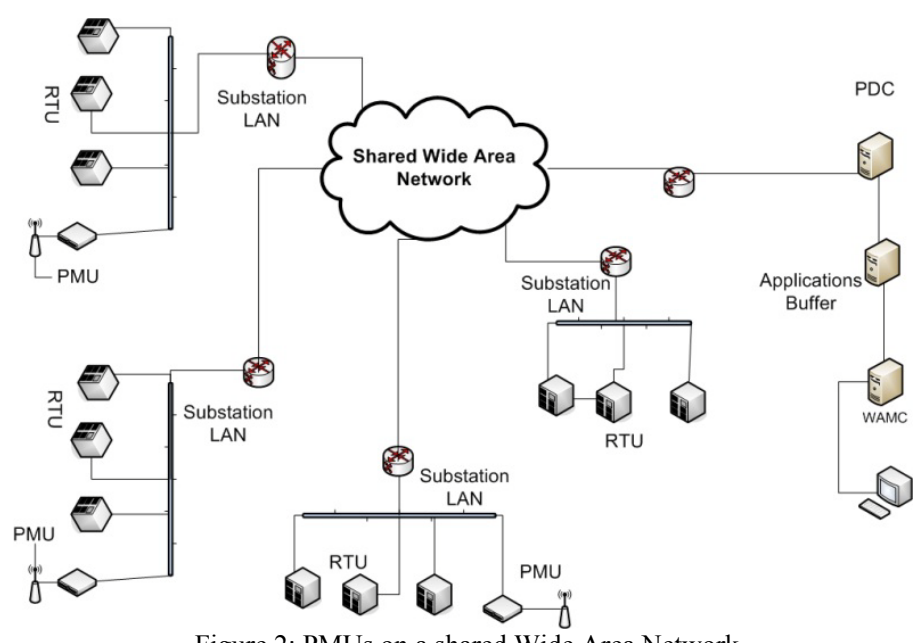

Figure 2: PMUs on a shared Wide Area Network

A note worth mentioning is that in using the TCP/IP suite for the higher network layers, the infrastructure (physical layer) can also be optical fiber. Besides, it is possible to utilize physical layer communication technologies such as microwave, co-axial, and radio. The main concern that arises in this case is reliability of the network infrastructure and the governing protocol suite, TCP/IP.

\section{Quality of Service in Power Communication Networks}

As mentioned earlier, the TCP/IP suite does not by itself guarantee the level of performance since it is a "best effort" protocol suite.

When the TCP/IP was designed, the main idea was to connect different parties in order to exchange textual information over a wide area network. However, this goal was set with the mentality to design a set or protocols to carry textbased data depending on the status of the network and traffic, 
while at the same time being designed for wire-line networks (i.e. without integrating wireless issues into consideration). The performance guarantee is solely left for the application developer to leverage the design in order to meet satisfactory power-network needs.

Consequently, it is essential to study how the TCP/IP technology should be used and in which ways "power-data" traffic shall be sent over the network in order to achieve the QoS goals. The main QoS parameters for TCP/IP are: bandwidth, delay (including but not limited to delay jitter), and packet loss. In order to enable power-network control to function in a satisfactory manner we investigate different responses of the network under study for different traffic models. In doing so, we can also understand the effect of the traffic shapes on QoS parameters, especially delay, which is the focus of our study. We consider this as the start of a research work, whose goal is to gather a large set of data on different traffic types and their corresponding response in power networks (carrying power information). When such a set is available it can form a standard for testing and for building more control algorithms for better QoS over Power Grid networks.

At the same time, two major QoS protocols are used in power-networks, namely Resource Reservation Protocol (RSVP) [11] and Multi-Protocol Label Switching (MPLS) [12]. RSVP is used for integrated services over a TCP/IP network. RSVP functions for the application data by requesting specific performance from the network for particular traffic-flow(s). RSVP is deployed on IP-routers with the purpose of leveraging QoS control.

MPLS also works on traffic flows between different nodes and between different applications. One interesting feature of MPLS is that is can function together with existing routing protocols. For instance, it can have an interface with RSVP. This makes the use of MPLS very attractive for our purpose. MPLS is an adaptable and many-sided neat protocol that can tackle issues related to network performance, scalability, and traffic flow.

\section{IMPLEMENTING PMU COMMUNICATION SCENARIOS}

In this section we describe the simulation models that were built to study the impact of QoS mechanisms on the PMU communication. The simulation models were built using OMNET++ an open source communication simulation environment [13]. The models were based on possible locations of PMUs in Sweden. The information required to build the models was acquired from interviews with experts at the Swedish TSO, previous surveys and case studies [14][15], this information included, possible location of PMUs in Sweden, number of RTUs, size and bandwidth allocation for RTU and PMU data packets, as well as the data rate for these PMUs. It is important to note while the simulation model does not represent the actual network, it does provide a good characterization of the Swedish TSO's Network and while this characterization is specific to the Swedish TSO the outcome of this study is nevertheless utilizable for other Transmission System Operators.

\section{A. Network Model}

Figure 3 provides a high level illustration of the network model that was built depicting the geographic range of the model.

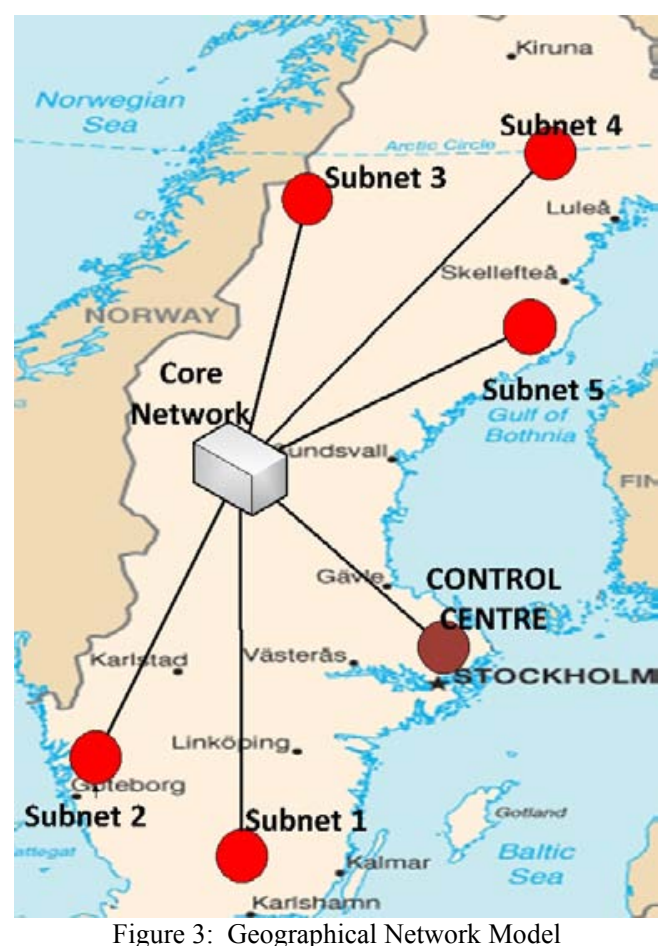

The high level model depicted in Figure 3 consists of three types of main subnets: the control center, core network and substation subnet. Subnets are a generalization of a portion of the network. Each subnet therefore may contain a several device models. The control center is the main subnet to which other nodes in other subnets in the network send information to. The core network therefore has a model of a SCADA Front end and a PDC. The front end receives packets from the RTU and the Video Streaming devices, while the PDC receives packets from the PMUs. The core network represents the core of the Wide Area Network and consists of a set of meshed router models. Finally, the subnets that represent substations contain models of PMUs, RTUs and Video streaming devices. Some substation subnets may actually contain more than one Substation and therefore more than one PMU.

\section{B. Network scenarios}

In order to examine and evaluate the network behavior under different characteristics and circumstances, the parameters in the simulation model was varied to create several scenarios. The variation resulted in to two scenario sets that cover PMU communication coming in from other devices such as RTUs and Video streaming devices. Since our main concern is the PMU communication the data coming from RTUs and Video devices is considered to be the background traffic and therefore has a lower priority over traffic coming from the PMUs. Each scenario set is made up of three scenarios in which they the difference between the scenarios is the bandwidth of the communication network and the existence of a QoS mechanism to differentiate the traffic 
on the network. Figure 4 below illustrates the common logical configuration for all scenarios.

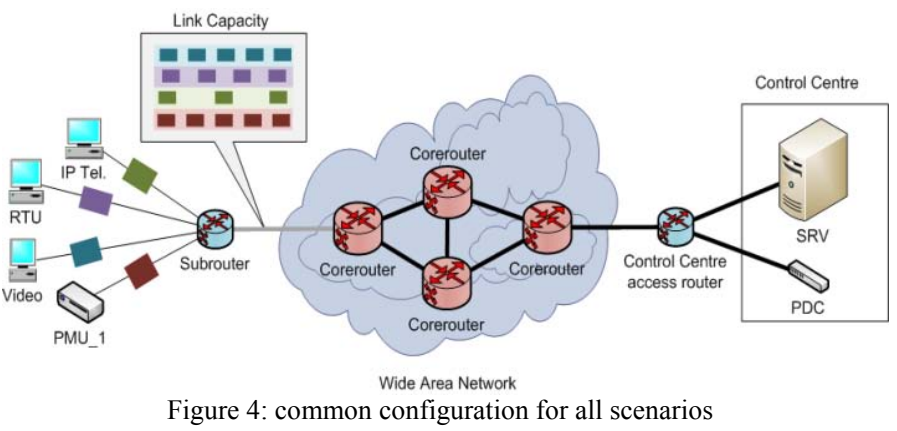

The bandwidth of the communication network between the substation and the control center was selected to be $2 \mathrm{Mbit} / \mathrm{s}$, 4Mbit/s and $8 \mathrm{Mbit} / \mathrm{s}$. In the first scenario set, QoS mechanisms were not implemented while in the second scenarios set, both RSVP and MPLS were implemented. The following Table 1 summarizes the scenarios and their configurations.

Table 1: Summery of Scenarios

\begin{tabular}{|c|c|c|c|c|c|c|}
\hline & Scenario & $\begin{array}{c}\text { Network } \\
\text { Bandwidth }\end{array}$ & QoS & PMUs & RTUs & $\begin{array}{c}\text { Video } \\
\text { Streaming }\end{array}$ \\
\hline \multirow{3}{*}{ Set 1 } & 1 & $2 \mathrm{Mbits} / \mathrm{s}$ & No & 8 & 200 & 2 \\
\cline { 2 - 7 } & 2 & $4 \mathrm{Mbits} / \mathrm{s}$ & No & 8 & 200 & 2 \\
\cline { 2 - 7 } & 3 & $8 \mathrm{Mbits} / \mathrm{s}$ & No & 8 & 200 & 2 \\
\hline \multirow{3}{*}{ Set 2 } & 4 & $2 \mathrm{Mbits} / \mathrm{s}$ & Yes & 8 & 200 & 2 \\
\cline { 2 - 7 } & 5 & $4 \mathrm{Mbits} / \mathrm{s}$ & Yes & 8 & 200 & 2 \\
\cline { 2 - 7 } & 6 & $8 \mathrm{Mbits} / \mathrm{s}$ & Yes & 8 & 200 & 2 \\
\hline
\end{tabular}

The payload for PMU devices was configured according to C37.118 protocol [16] to be 40 bytes long assuming that each packet consisted of one Phasor sample. Table 2 summarizes data rates, payloads, reserved link capacity and different protocol header sizes for each of the data flows used during the simulations as well as the traffic generated by each unit.

Table 2 - Data Rate, Packet Size and Generated Traffic for Data Flows for nodes in all scenarios.

\begin{tabular}{|c|c|c|c|c|}
\hline Data flow & $\begin{array}{l}\text { Data Rate } \\
\text { (Packet/s) }\end{array}$ & $\begin{array}{c}\text { Payload } \\
\text { (bytes) }\end{array}$ & $\begin{array}{l}\text { Headers } \\
\text { UDP/IP }\end{array}$ & $\begin{array}{c}\text { Generated } \\
\text { traffic per unit }\end{array}$ \\
\hline PMU & 30 & 40 & 28 & $19.2 \mathrm{Kbit} / \mathrm{s}$ \\
\hline RTU & 2 & 500 & 28 & $8 \mathrm{Kbit} / \mathrm{s}$ \\
\hline Video & 200 & 1024 & 28 & $1.7 \mathrm{Mbit} / \mathrm{s}$ \\
\hline
\end{tabular}

\section{TCP and UDP}

As discussed in the section II. , since RSVP and MPLS can work together in providing high reliability and accuracy in data delivery, the UDP over an IP network is used as a transport-layer protocol for all the data flows, including the PMU data flow, in all scenarios (as shown in Table 2).

Since we aim at minimizing delay and packet loss to achieve a power-application satisfactory limit, we firstly discuss which Transport protocol is best suitable. The two protocols to consider are TCP and UDP (User Datagram Protocol). TCP has relatively large headers compared to UDP [17]. Moreover, the control mechanisms and algorithms of TCP utilize some bandwidth and impose some delays such as the "TCP slow start" that is used when a packet loss occurs. Such algorithms in TCP make it not attractive for our type of application since TCP is itself "best effort" also.
On the other hand, UDP is a connectionless protocol, i.e. it does not apply no control over the transmission and reception of packets except for data checksum (that is needed in any transaction) to insure reliability and correctness of the received sequence of bits in the whole IP packet. In brief, the UDP checksum runs the check over the whole IP packet, however, when a packet is lost, UDP does not re-transmit the packet. Therefore, UDP does not input any new bytes (traffic) on the network and when a packet is lost. This is attractive for our application from the point of view that no extra and unknown packets (nor acknowledgements) are sent over the network. However this is not suitable to guarantee any level of QoS. A major factor that influences this choice is the type of data, which is sent. Depending on the requirements for the data, few required characteristics can be established. In brief, the trade-off between these two protocols is that between speed and reliability. UDP counts as a less reliable but fast protocol, whereas TCP is a more reliable but also a slower protocol [18].

However, to make the best use of UDP in our application, other protocols shall be used to ensure minimum performance, this requirements is possible by using RSVP and MPLS along with UDP.

On the other hand, one of the important parameters to study, when the aforementioned protocols are used, is the whole endto-end delay. This is because if delay is only improved along one or only few hops on a path, this does not guarantee that the other remaining hops would act in a way that suits the overall delay limit that shall be respected throughout the communication. This is the reason why, in the delay issue, we focus on the end-to-end delay from the source to destination over a network-path,

In Scenario 1, 2 and 3 there are no QoS schemes implemented, thus using UDP as the transport protocol is not the best choice, but it presents the opportunities to compare how QoS schemes upgrade the network performance. In case of an eventual congestion in the network, it is of outermost importance that PMU data is not completely lost and because of the lack of any QoS schemes in scenarios set 1. In Scenario 4,5 and 6 , UDP is also used as a communication protocol for the PMU data flow. The main reason is the speed-advantages it provides. The reliability issue is now solely dependent on QoS where congestion is avoided through bandwidth reservation and prioritization. Another issue which might present itself by using TCP is the fact that TCP-sessions are created and shut down during a transmission. This process depends on what kind of data flow is using the TCP protocol. In the case of PMU data flow where the flow is continuous the TCP-session will be shut down when it is timed out or if a unit/link breaks down. This in turn will result in the creation of a new session, and if this is the case of numerous PMUs, it might cause congestion at the server site.

\section{SimUlATiOn RESUlts}

End-To-End (ETE) latency and the packet loss regarding the PMU traffic were collected from the simulations. The ETE latency of a certain data flow is measured by calculating the time it takes for every packet to reach its destination. This calculation is done by following the formula stated below: 


$$
\text { Latency }_{n}=\text { Arrival time }_{n}-\text { Sending time } e_{n}
$$

Equation (1) reads that by subtracting the packet sending time from its arrival time, the packet latency is obtained.

Packet loss on the other hand is measured by subtracting the total amount of packets sent by the PMU from the total amount of PMU packets that were actually received by the PDC, this gives the percentage overall packet loss.

\section{A. End-To-End Latency Results}

The results obtained for the ETE latency in scenario sets 1 and 2 are presented in Figure 5 and Figure 6 respectively. In scenario set 1 , the peak values for the ETE latency are $46 \mathrm{~ms}$ with $2 \mathrm{Mbit} / \mathrm{s}$ network capacity, $10.6 \mathrm{~ms}$ with $4 \mathrm{Mbit} / \mathrm{s}$ network capacity and $6.4 \mathrm{~ms}$ when the total network capacity is $8 \mathrm{Mbit} / \mathrm{s}$.

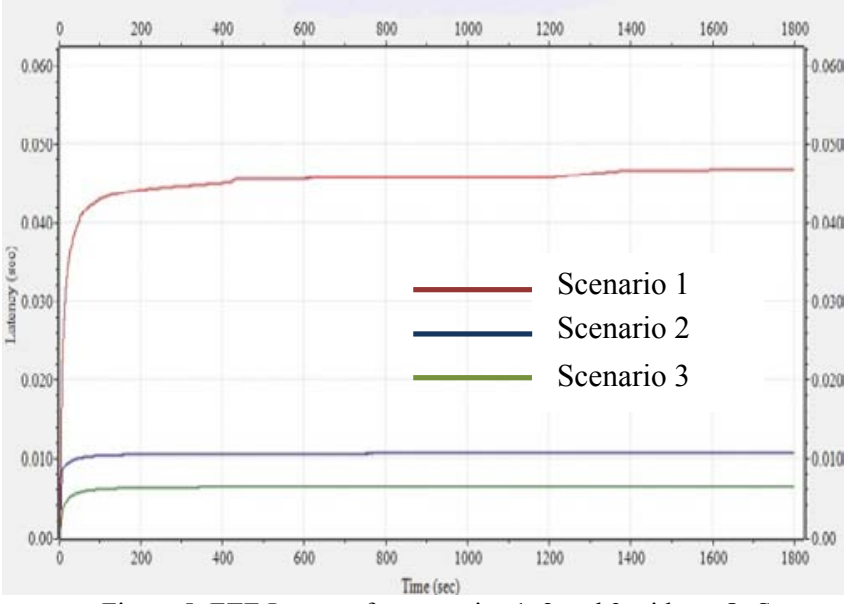

Figure 5: ETE Latency for scenarios 1, 2 and 3 without QoS.

In scenario set 2, the respective latency values for 2,4 and 8 $\mathrm{Mbit} / \mathrm{s}$ network capacity are $12 \mathrm{~ms}, 10 \mathrm{~ms}$ and $6.4 \mathrm{~ms}$. It can be noticed that the latency does not vary drastically between the scenarios apart from the $2 \mathrm{Mbit} / \mathrm{s}$ simulations, where the difference is $34 \mathrm{~ms}$. This deviation depends on the network instability and the lack of packet prioritization at the routers. When analyzing the results from each scenario separately it is evident that the decrease of network capacity results with higher ETE latency

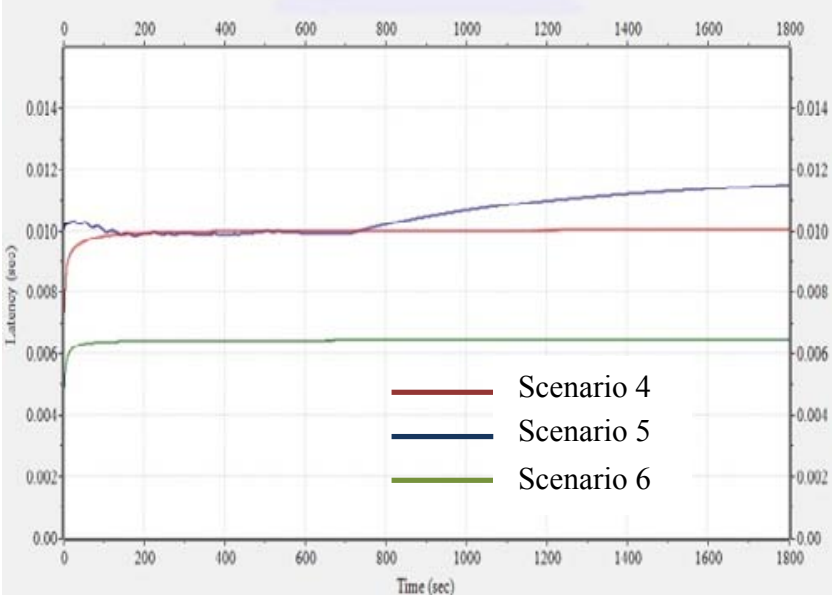

Figure 6: ETE Latency for scenarios 4, 5 and 6, with QoS,

\section{B. Packet Loss}

The packet loss rates that were measured during the simulations of scenario sets 1 and 2 are summarized in Figure 7. In scenario set 1, with $2 \mathrm{Mbit} / \mathrm{s}$ network capacity, the packet loss rate for the PMU packets is $69.4 \%$. For the same scenario, with $4 \mathrm{Mbit} / \mathrm{s}$ and $8 \mathrm{Mbit} / \mathrm{s}$ total network capacity, the packet loss values are $21.9 \%$ and $13.6 \%$ respectively.

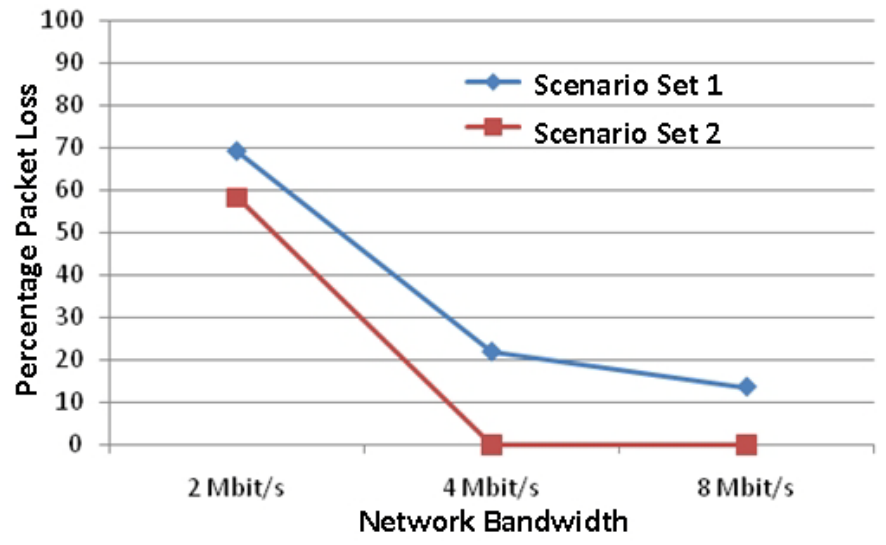

Figure 7: Percentage packet loss vs. network bandwidth for scenarios $1 \& 2$.

The high packet loss rates depend on the lack of sufficient network capacity and packet prioritization. In scenario set 2, with total network capacity of $2 \mathrm{Mbit} / \mathrm{s}$, the packet loss is 58.3 $\%$. The corresponding packet loss values measured with 4 Mbit/s and $8 \mathrm{Mbit} / \mathrm{s}$ network capacity, are $0.03 \%$ and $0.028 \%$ respectively. The high packet loss in the $2 \mathrm{Mbit} / \mathrm{s}$ simulation depends on the insufficient network capacity compared to the total amount of generated traffic, approximately 3 times more than the available network capacity. However, the benefits of low packet loss provided by the implementation of RSVP and MPLS are evident in the latter simulations. In the second simulation, even though the generated traffic exceeds the network capacity of $4 \mathrm{Mbit} / \mathrm{s}$, the PMU packet loss is still reasonably low due to the sufficient amount of reserved bandwidth in the network and the presence of packet prioritization. The results for ETE latency and packet loss, measured in both scenario sets are summarized in Table 3 .

Table 3 - Summery of Simulation Results

\begin{tabular}{|c|c|c|c|c|c|c|}
\hline Scenario & \multicolumn{2}{|c|}{$2 \mathrm{Mbit} / \mathrm{s}$} & \multicolumn{2}{c|}{$4 \mathrm{Mbit} / \mathrm{s}$} & \multicolumn{2}{c|}{$8 \mathrm{Mbit} / \mathrm{s}$} \\
\cline { 2 - 7 } & Latency & $\begin{array}{c}\text { Packet } \\
\text { Loss }\end{array}$ & Latency & $\begin{array}{c}\text { Packet } \\
\text { Loss }\end{array}$ & Latency & $\begin{array}{c}\text { Packet } \\
\text { Loss }\end{array}$ \\
\hline $\begin{array}{c}\text { Scenario } \\
\text { Set 1 }\end{array}$ & $46 \mathrm{~ms}$ & $\begin{array}{c}69.4 \\
\%\end{array}$ & $10.6 \mathrm{~ms}$ & $\begin{array}{c}21.9 \\
\%\end{array}$ & $6.4 \mathrm{~ms}$ & $\begin{array}{c}13.6 \\
\%\end{array}$ \\
\hline $\begin{array}{c}\text { Scenario } \\
\text { Set 2 }\end{array}$ & $12 \mathrm{~ms}$ & $\begin{array}{c}58.3 \\
\%\end{array}$ & $10 \mathrm{~ms}$ & $\begin{array}{c}0.03 \\
\%\end{array}$ & $6.4 \mathrm{~ms}$ & $\begin{array}{c}0.028 \\
\%\end{array}$ \\
\hline
\end{tabular}

\section{Assumptions}

Even though the positive results from the simulations, there are some assumptions that have been made. The assumptions have been drawn regarding queue sizes in routers, packet sizes and transmission rates for RTU- and IP Telephony traffic. However, the respective packet sizes and transmission rates for RTU and IP Telephony are in this case not so decisive as long as they generate the desired amount of background traffic in the network. 


\section{CONCLUSIONS}

The presented investigation shows how the usage of QoS schemes, in particular RSVP and MPLS, can improve powernetwork performance. In order to accomplish a relevant and practical study on varied network environments, several network scenarios are created based on real industrial needs and requirements. The diversity in the scenarios enabled comparisons between simulation results, which in turn lead to a more complete analysis of the network performance.

The most important metrics are the ETE latency and packet loss for PMU packets. The results acquired for the ETE latency are well below the established requirements. This in turn indicates that the current network configuration can handle the present amount of traffic. An Improvement can also be seen in the results for packet loss. Clearly the QoS schemes used in the simulations also keep the packet loss rates low and thereby contributes to an overall performance improvement. A critical factor for the positive simulation results, although the increased amount of traffic in the network (Scenarios 3, 4 and 5 ) is the use of RSVP. Hence, integrating additional PMUs in a network, while keeping the network performance within acceptable boundaries, is possible as long as adequate amount of bandwidth is reserved throughout the link paths.

However, to maintain low packet loss values shows to be a bit more demanding. The usage of RSVP and packet prioritization is not solely enough, but the overall link capacity needed is at least $4 \mathrm{Mbit} / \mathrm{s}$ in order to obtain acceptable packet loss rates.

\section{REFERENCES}

[1] A. Phadke, "The Wide World of Wide-Area Measurements" IEEE Power and Energy Magazine, Vol. 2, No. 4, September /October 2008. pp 52-65.

[2] D. Karlsson, M. Hemmingsson, S. Lindahl, "Wide Area System Monitoring and Control", IEEE Power \& Energy Magazine, Vol. 2, No. 5, September/October 2004, pp 68-76.

[3] M. Zima, M. Larsson, P. Korba, C. Rehtanz, G. Andersson; "Design Aspects for Wide- Area Monitoring and Control Systems"; Proceedings of the IEEE, Vol. 93, No. 5, May 2005, pp .980 - 996.

[4] A.B Leirbukt, J.O Gjerde, P. Korba, K. Uhlen, L. K Vormedal, L. Warland, "Wide Area Monitoring Experiences in Norway," Power Systems Conference and Exposition, 2006. PSCE '06. 2006 IEEE PES , vol., no., pp.353-360, Oct. 29 2006-Nov. 12006.

[5] North American Synchrophasor Initiative, www.naspi.org.

[6] D. Bakken, C. H. Hauser, H. Gjermundrod, A. Bose. "Towards More Flexible and Robust Data Delivery for Monitoring and Control of the Electric Power Grid", Technical Report EECS-GS-009, School of Electrical Engineering and Computer Science, Washington State University, May 30, 2007. http://www.gridstat.net/publications/TR-GS009.pdf.

[7] C. Marinez, M Parashar, J. Dyer, J. Coroas."Phasor Data Requirements for Real Time Wide-Area Monitoring, Control and Protection Applications" CERTS/EPG, EIPP-Real Time Task Team. January 2005. Retrieved from: http://www.naspi.org/resources/archive/rttt/eipp_ realtime_group_data_requirements_draft5_jan26_2005.pdf.

[8] M. Kim, M.J. Damborg, J. Huang, S.S Venkata. "Wide-Area Adaptive Protection Using Distributed Control and High-Speed Communication" Proceedings of the 14th power system computation conference (PSCC), 2002.

[9] B. Naduvathuparambil, M. C. Valenti, A. Feliachi. "Communication Delays in Wide Area Measurement Systems" Proceedings of the ThirtyFourth Southeastern Symposium on System Theory, 2002.

[10] K. H Mak., B.L Holland., "Migrating electrical power network SCADA systems to TCP/IP and Ethernet networking," IEE Power Engineering Journal, vol.16, no.6, pp. 305-311, Dec 2002
[11] R. Braden, L. Zhang, S. Berson, S. Herzog, S. Jamin, "Resource ReSerVation Protocol (RSVP), Version 1, Functional Specification", Network Working Group, Request for Comments (RFC) 2205, Standards Track Category, September 1997.

[12] E. Rosen, Y. Rekhter, "BGP/MPLS VPNs," Network Working Group, Request for Comments (RFC) 2547, Informational Category, March 1999.

[13] OMNET++, http://www.omnetpp.org/

[14] M. Chenine, E. Karam, L. Nordström, "Modeling and Simulation of Wide Area Monitoring and Control Systems in IP-based Networks". In the Proceeding of the IEEE PES General Meeting 2009. July 2009

[15] M. Chenine, K. Zhu, L. Nordström. "Survey on Priorities and Communication Requirements on PMU-based Applications in the Nordic Region" In the Proceedings of IEEE Power Tech 2009. June 2009.

[16] IEEE standard for Synchrophasors for Power Systems. IEEE Standard C37.118-2005. March 2006.

[17] W. R. Stevens, "TCP IP Illustrated”, Vol. 1, Addison-Wesley, 1994.

[18] T. Kenyon. "High Performance Data Network Design". ButterworthHeinemann Digital Press, 2002. 